\title{
A pooled analysis of the outcome of prospective colonoscopic surveillance for familial colorectal cancer
}

\author{
David Mesher ${ }^{1}$, Isis Dove-Edwin ${ }^{2}$, Peter Sasieni ${ }^{1}$, Hans Vasen ${ }^{3,4}$, Inge Bernstein ${ }^{5,6}$, Brigitte Royer-Pokora ${ }^{7}$, \\ Elke Holinski-Feder ${ }^{8,9}$, Fiona Lalloo ${ }^{10}$, D. Gareth Evans ${ }^{10}$, Anna Forsberg ${ }^{11}$, Annika Lindblom ${ }^{11}$ and Huw Thomas ${ }^{2}$ \\ ${ }^{1}$ Queen Mary University of London, Centre for Cancer Prevention, Wolfson Institute of Preventive Medicine, Charterhouse Square, London, United Kingdom \\ ${ }^{2}$ St Mark's Hospital, Imperial College London, London, United Kingdom \\ ${ }^{3}$ The Netherlands Foundation for the Detection of Hereditary Tumours, Leiden, The Netherlands \\ ${ }^{4}$ Department of Gastroenterology and Hepatology, Leiden University Medical Centre, Leiden, The Netherlands \\ ${ }^{5}$ The Danish HNPCC-register, Department of Gastroenterology and Clinical Research Center, Copenhagen University Hospital, Hvidovre, Denmark \\ ${ }^{6}$ The Surgical Gastroenterology Department, Aalborg University Hospital, Aalborg, Denmark \\ ${ }^{7}$ Institute of Human Genetics and Anthropology, Heinrich Heine University, Medical Faculty, Duesseldorf, Germany \\ ${ }^{8}$ Klinikum der Universität München, Campus Innenstadt, Medizinische Klinik und Poliklinik IV, Ziemssenstr. 1, 80336, Munich, Germany \\ ${ }^{9}$ MGZ - Medizinisch Genetisches Zentrum, Bayerstr. 3-5, 80335, Munich, Germany \\ ${ }^{10}$ Genetic Medicine, Manchester Academic Health Science Centre, St Mary's Hospital, Manchester, United Kingdom \\ ${ }^{11}$ Department of Molecular Medicine and Surgery, Karolinskalnstitutet, Stockholm, Sweden
}

Surveillance guidelines for the management of familial colorectal cancer (FCC), a dominant family history of colorectal cancer in which the polyposis syndromes and Lynch syndrome have been excluded, are not firmly established. The outcome of colonoscopic surveillance is studied using data from six centers. DNA mismatch repair deficiency was excluded by genetic testing. Families were classified as FCC type $\mathrm{X}$ if they fulfilled the original Amsterdam criteria (AC) and late onset (LOFCC) if they fulfilled the AC apart from not having a cancer aged under 50. The most advanced findings on colonoscopy were analyzed. One thousand five hundred eighty-five individuals (median age 47.3, 44\% male) from 530 FCC families (349 FCC type X) underwent a total of 4,992 colonoscopies with 7,904 patient-years of follow-up. Results for FCC type X and LOFCC were very similar. At baseline, 22 prevalent asymptomatic colorectal cancers were diagnosed, 120 (7.6\%) individuals had high-risk adenomas and 225 (14.2\%) simple adenomas. One thousand eighty-eight individuals had a further colonoscopy (median follow-up of 6.2 years). Of nine individuals diagnosed with cancer, eight had a previous history of at least one polyp/adenoma. High-risk adenomas were detected in $92(8.7 \%)$ and multiple adenomas were detected in $20(1.9 \%)$ individuals. Both FCC type X and LOFCC have a high prevalence of colorectal cancers and on follow-up develop high-risk adenomas (including multiple adenomas), but infrequent interval cancers. They should be managed similarly with five-yearly colonoscopies undertaken from between 30 and 40 with more intensive surveillance in individuals developing multiple or high-risk adenomas.

Approximately, 3-5\% of all cases of colorectal cancer (CRC) are associated with a highly-penetrant, dominantly inherited syndrome. These syndromes may be identified due to a strong family history of CRC and, by their phenotype or by germline genetic testing, and their clinical management is firmly established. ${ }^{1,2}$ The most common syndrome is Lynch syndrome (LS) (previously known as hereditary nonpolyposis colorectal cancer-HNPCC). LS is caused by an inherited alteration of DNA mismatch repair genes ( $M L H 1, M S H 2$, MSH6 or PMS2), which results in DNA microsatellite instability (MSI) in the tumor. Familial adenomatous polyposis and the hamartomatous syndromes are rarer. Familial

Key words: colonoscopic surveillance, familial colorectal cancer type X, Amsterdam criteria, pooled analysis

Abbreviations: AC: Amsterdam criteria; CRC: colorectal cancer; FAP: familial adenomatous polyposis; FCC: familial colorectal cancer; HNPCC: hereditary non-polyposis colorectal cancer; LOFCC: late onset familial colorectal cancer; LS: Lynch syndrome; MSI: micro-satellite instability; MSS: micro-satellite stability; NHS: National Health Service

Grant sponsor: The German HNPCC Consortium: Deutsche Krebshilfe; Grant number: German Cancer Aid 108252; Grant sponsor:

Central Manchester University Hospitals NHS Foundation Trust: Manchester BRC; Grant sponsor: St Marks Hospital Cancer Research UK Family Cancer Clinic: Cancer Research UK Imperial Cancer Centre; Grant number: CR-UK C37990/A12196; Grant sponsor: Queen Mary University of London; Grant number: CR-UK grant number C8162/A10406

DOI: $10.1002 / \mathrm{ijc} .28397$

History: Received 16 Apr 2013; Accepted 10 July 2013; Online 30 Jul 2013

Correspondence to: Professor Huw Thomas, Family Cancer Clinic, Imperial College London, St Mark's Hospital, Watford Road, Harrow, HA1 3UJ, United Kingdom, Tel.: + [44-(0)-208-235-4266], Fax: + [44-(0)-208-235-4277], E-mail: huw.thomas@imperial.ac.uk 


\section{What's new?}

About 3-5 percent of colorectal cancer cases are associated with a highly penetrant dominant inherited syndrome. However, established guidelines for the surveillance of Familial Colorectal Cancer (FCC), in which the Polyposis syndromes and Lynch syndrome have been excluded, are lacking. This study suggests that FCC and late-onset FCC (LOFCC) patients should be managed with five-yearly colonoscopies between ages 30 and 40 , with more intensive surveillance in individuals who develop multiple or high-risk adenomas. Little evidence was found to support intensive screening before age 30.

colorectal cancer (FCC) refers to the larger number of families who have dominantly inherited CRC but no polyposis phenotype and no evidence of altered DNA mismatch repair genes. Families who fulfill the three Amsterdam Criteria I for HNPCC [ $i$ ) At least three relatives over two generations affected with a CRC one of whom is a first-degree relative of the other two, (ii) FAP excluded, (iii) one CRC diagnosed aged under $50^{2}$ ], are referred to as FCC type X, and families who fulfill the criteria, except that they have no relatives diagnosed with CRC under the age of 50 years, are referred to as Late Onset FCC (LOFCC).

Lindor et al. have investigated the phenotype of FCC type $\mathrm{X}$ families and have shown that there is no increased incidence of the extra-colonic cancers (endometrial, ovarian, stomach, brain and small intestine) associated with LS and that the risk of CRC appears to be less than in LS. ${ }^{3}$ Authors of this study have previously undertaken studies on the outcome of colonoscopic surveillance in FCC type X families. ${ }^{4-7}$ Nevertheless, there is still a paucity of prospective evidence as to how the risk of CRC should be managed in FCC. The aim of this study was to use data pooled from six European centers to evaluate the outcome of prospective colonoscopic surveillance of individuals from FCC families to see whether recommendations for surveillance could be made based on the results. The protocols for surveillance vary between the centers and thus provide some information on the relative benefit of different age at initial surveillance and different intervals between surveillance colonoscopies.

\section{Material and Methods}

We approached European centers that have prospectively collected colonoscopic surveillance data from individuals from families at a high risk of CRC. The data presented are from six centers; Central Manchester University Hospitals NHS Foundation Trust, Manchester, UK; German Consortium for Hereditary Non-Polyposis Colorectal Cancer, Germany; The Danish HNPCC-register, Copenhagen University Hospital, Hvidovre, Denmark; Karolinska University Hospital, Stockholm, Sweden; The Netherlands Foundation for the Detection of Hereditary Tumours, Leiden, The Netherlands and St Mark's Hospital, London, UK.

Data were collected for individuals from FCC families. Patients eligible for the study were the first-degree, at-risk relatives of the defining $\mathrm{CRC}$ triad undergoing colonoscopic surveillance. The group was divided into FCC type X and LOFCC. Individuals from families with known germline LS mutations were not eligible.
DNA mismatch repair deficiency was excluded in the families by one of two methods in each of the centers and families were defined into two groups:

- Tumor testing group: One or more CRC tumors tested within each pedigree of which $>50 \%$ tumors are microsatellite stable (MSS) (Bat 26 sufficient) and/or do not demonstrate loss of expression of the DNA mismatch repair genes (MLH1, MSH2, MSH6 or PMS2) using immunohistochemistry.

- Germline testing group: families in whom only germline sequencing of at least the DNA mismatch repair genes MLH1 and MSH2 has been undertaken and no mutations detected.

For all eligible patients, data were collected on: (i) family history (Amsterdam Criteria I/family history, details of any metachronous and/or synchronous colorectal lesions in any defining triad member, details of any individual with a cancer (colorectal and extra colonic) found within the family including their sex, age, DNA MSI testing, date of birth, date of death, site of cancer, stage of cancer); (ii) patients undergoing colonoscopy (age, sex, date of birth, date of death); (iii) colonoscopy details for each colonoscopy performed (date, result, details of any tissue removed including histology, site, size, hyperplastic polyp, adenoma (tubular or villous), grade of dysplasia and whether colonoscopy was complete to cecum).

Pseudonymized data from each center were sent retrospectively to DM and data were reformatted and pooled to a single secure database. Data sent and details of each center are shown in Table 1.

The baseline colonoscopy was defined as the first colonoscopy undertaken in an individual. All subsequent colonoscopies are considered to be in the follow-up period of surveillance. We identified all cancers found at the baseline colonoscopy (or due to immediate follow-up of an abnormal baseline colonoscopy). If the baseline colonoscopy was performed due to symptoms, cancers were considered to be symptomatic and excluded from both the baseline analysis and any follow-up analysis. Otherwise, any cancer detected was considered to be "(asymptomatic), prevalent screen detected" and the results of further surveillance for these individuals were excluded from the follow-up analysis.

The endpoints of the study are presence of adenomas or cancer. Multiple adenomas were defined as an occurrence of three or more adenomas on any one colonoscopy, five or more adenomas by the age of 50 years, eight or more by age 60,11 or more by age 70 or 14 or more adenomas over total 

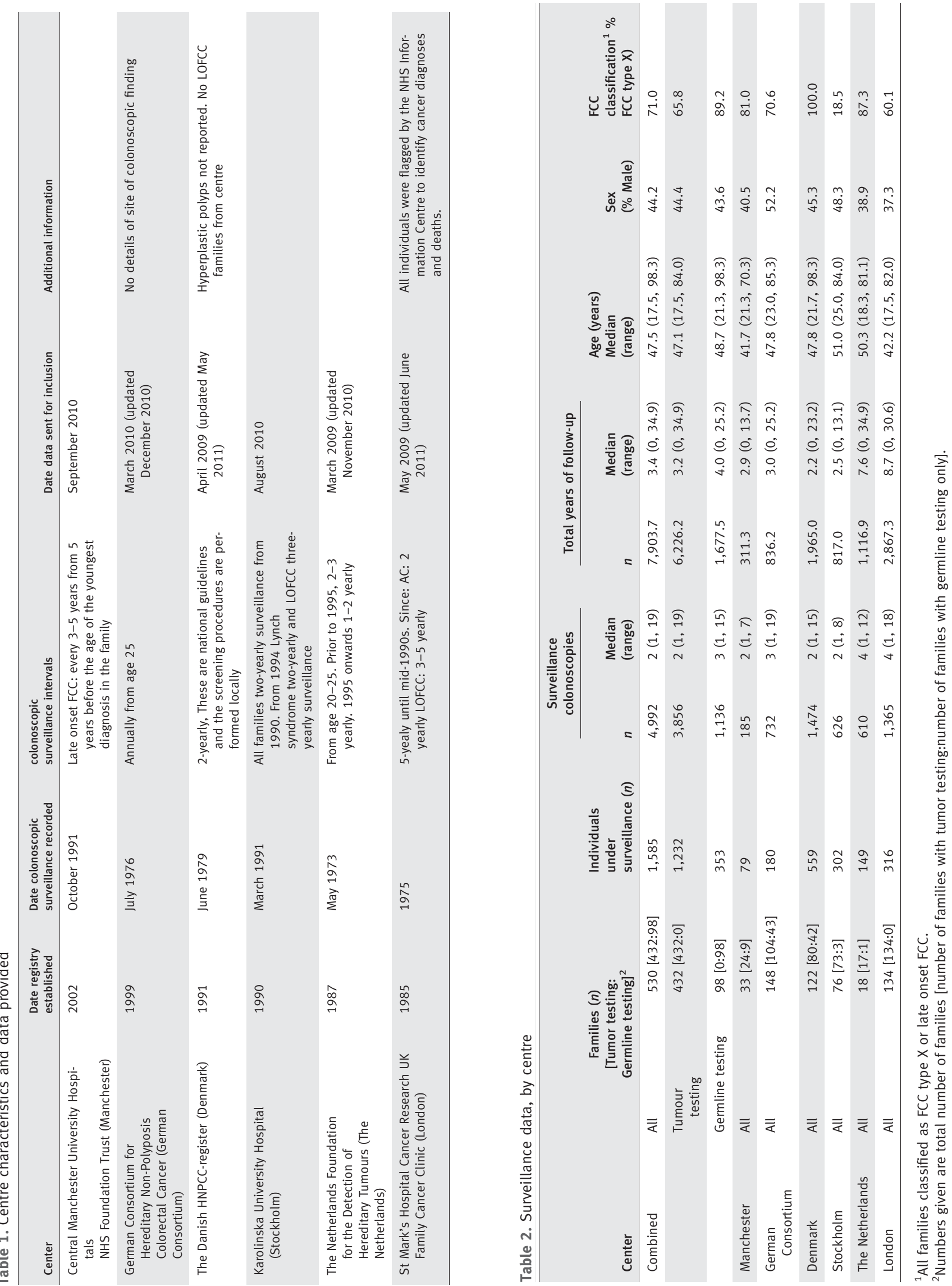
surveillance period. High-risk adenomas were defined as an adenoma with villous histology or a diameter $>10 \mathrm{~mm}$ or high-grade dysplasia.

The pooled analysis of pseudonymized data is considered to be service evaluation and did not require ethical approval in the UK. Ethical approval was obtained locally, where required, for the original data collection.

\section{Statistical methods}

Total surveillance period is taken from the time of the baseline colonoscopy to the last colonoscopy (excluding those after the diagnosis of $\mathrm{CRC)}$ for each individual under surveillance.

We initially report the most advanced finding on the baseline colonoscopy for each individual (Normal, Hyperplastic polyp(s), Adenoma(s), High-risk adenoma, CRC). This result is tabulated against familial phenotype. Percentages are weighted to standardize by age group to allow for differences in the age at first screen between individuals from FCC type $\mathrm{X}$ and LOFCC families.

Using published data from the Flexi-sig trial, ${ }^{8}$ we estimated the number of distal cancers detected soon after randomization in the screened arm to be approximately equal to the number of distal cancers diagnosed within 4.0 years of randomization in the control arm. Assuming the lead-time distribution and sensitivity of endoscopy in this study was similar to that in the Flexi-sig trial, the number of CRCs found in the baseline colonoscopy here would be similar to the number expected over 4 years in the absence of screening. Had our cohort had average population risk, the "expected" number of incident cancers in the four years following baseline colonoscopy was calculated using CRC rates from England for 2009 stratified by sex and 5-year age group. ${ }^{9}$

The most advanced pathological finding on any subsequent colonoscopy is presented according to the baseline colonoscopy result.

Rates of (first) adenoma, high-risk adenoma and CRC (dependent on age, sex, familial phenotype and previous findings) are calculated with individuals censored at the first event (as this may prevent the subsequent development of high-risk adenomas or cancers). Events found at a colonoscopy are assumed to have developed between the previous colonoscopy and the diagnosing colonoscopy.

In order to estimate the cumulative proportion of subjects at different ages with a particular event (adenoma/multiple adenomas/high-risk adenoma/CRC), we consider the time to first event in an individual. For each individual and each event type, we consider the last colonoscopy before an event was found and the first colonoscopy on which an event was found. The data are interval censored as the date of an event is only known to be at some point since an individual's previous colonoscopy. For individuals without an event, we consider only their last colonoscopy. Similarly, only the first is used for an individual with an event found at baseline. As event times are either left-censored or right censored (never observed exactly), the Kaplan-Meier estimates are not appropriate, hence the proportion of colonoscopies with an event is estimated by smoothing (we used the Stata command lowess which is similar to a moving average) and the result is forced to be non-decreasing in age by applying the pool adjacent violators algorithm. ${ }^{10}$

\section{Results}

The outcome of colonoscopic surveillance was collected for 1,585 at-risk individuals from 530 FCC families from the six European centers. A total of 4,992 colonoscopies have been undertaken with 7,904 person-years of follow-up.

DNA mismatch repair deficiency was excluded by testing one or more cancer for MSI and/or immunohistochemistry in $81.5 \%$ of families and by germline genetic testing of MLH1 and MSH2 alone in $18.5 \%$ of families. The method used varied between centers between $66 \%$ and $100 \%$ of families classified by tumor testing at each center. The median number of colonoscopies per individual ranged from 2 to 4 across centers and median total follow-up ranged from 2.2 years to 8.7 years. Age at baseline colonoscopy and the proportion of males was similar across centers with median values of 47.5 years and 44\%, respectively. Full details are shown in Table 2.

Of 1,585 individuals under surveillance, 1,126 (71\%) were from families classified as FCC type X and 459 (29\%) from LOFCC with a total of 3,790 and 1,202 surveillance colonoscopies, respectively. Median follow-up was slightly higher amongst individuals from FCC type X families compared to LOFCC (median (range) follow-up time: 4.1 years $(0,30.6)$ and 3.0 years $(0,34.9)$ respectively).

\section{Findings at baseline colonoscopy}

In total, there were $44(2.8 \%)$ cancers that were identified either at the baseline screen or on further investigation of an abnormality detected at baseline colonoscopy. The age at cancer diagnosis ranged from 31 to 79 years (median age 51.5 years). Twenty-two cancers were diagnosed in individuals who were symptomatic (e.g., change in bowel habit, rectal bleeding or anemia) at the time that they were referred for colonoscopy and these cases were excluded from analysis. In the remaining 22 individuals, cancers were classified as asymptomatic prevalent cancers. Based on data of CRC diagnoses in England in 2009, ${ }^{9}$ we calculated an expected number of prevalent cancers on the baseline colonoscopy of 4.08. Relative to this, the 22 asymptomatic prevalent cancers yield a (standardized prevalence) ratio of 5.4 (95\% CI 3.4-8.2) compared to the general population. Of the 44 cancers, 32 were from the left side of the colon and 11 from the right (there were slightly more cancers from the left side of the colon amongst those with symptoms than those who were asymptomatic: $86 \%$ vs. $64 \%$, respectively).

At least one adenoma was reported in 345/1,585 (21.8\%) individuals and, of these, 120/345 (34.8\%) were categorized as high-risk adenomas. The age at first screen amongst 
Table 3. Worst result at baseline for each individual (by subgroup). Numbers shown are $\mathrm{n}$ (age standardized percentage)

\begin{tabular}{llll}
\hline & \multicolumn{3}{c}{ Subgroup } \\
\cline { 2 - 4 } Worst result & FCC type $X$ & Late onset FCC & Total \\
\hline Normal & $760(66.8)$ & $256(57.6)$ & $1016(64.1)$ \\
No histology recorded & $28(2.5)$ & $10(2.1)$ & $38(2.4)$ \\
Metaplastic/Hyperplastic Polyp & $96(8.5)$ & $68(14.7)$ & $164(10.3)$ \\
Adenoma(s) & $151(13.9)$ & $74(15.3)$ & $225(14.2)$ \\
High risk Adenoma ${ }^{1}$ & $75(6.9)$ & $45(9.3)$ & $120(7.6)$ \\
Colorectal cancer & $16(1.5)$ & $6(1.1)$ & $22(1.4)$ \\
Total & $1126(100)$ & $459(100)$ & $1585(100)$ \\
\hline
\end{tabular}

${ }^{1}$ High Risk Adenoma is defined as an adenoma with villous histology or a diameter of $10 \mathrm{~mm}$ or greater or high-grade dysplasia.

Table 4. Initial result vs. worst colonoscopic result after initial colonoscopy amongst FCC type X families

\begin{tabular}{llllllll}
\hline & \multicolumn{7}{c}{ Worst follow-up result } \\
\cline { 2 - 7 } Worst baseline result & Normal & $\begin{array}{l}\text { Hyperplastic } \\
\text { Polyp }\end{array}$ & Adenoma & $\begin{array}{l}\text { Multiple } \\
\text { adenomas }\end{array}$ & $\begin{array}{l}\text { High Risk } \\
\text { adenoma }\end{array}$ & Cancer & Total \\
\hline Normal & $326(63.8)$ & $54(10.6)$ & $91(17.8)$ & $6(1.2)$ & $32(6.3)$ & $2(0.4)$ & $511(100)$ \\
Hyperplastic Polyp & $19(25.3)$ & $25(33.3)$ & $14(18.7)$ & $7(9.3)$ & $9(12.0)$ & $1(1.3)$ & $75(100)$ \\
\hline Adenoma & $38(36.5)$ & $10(9.6)$ & $36(34.6)$ & $7(6.7)$ & $12(11.5)$ & $1(1.0)$ & $104(100)$ \\
Multiple adenomas & $3(18.8)$ & $2(12.5)$ & $1(6.3)$ & $2(12.5)$ & $6(37.5)$ & $2(12.5)$ & $16(100)$ \\
High risk adenoma & $24(40.7)$ & $2(3.4)$ & $11(18.6)$ & $1(1.7)$ & $21(35.6)$ & - & $5(100)$ \\
Total & $410(53.6)$ & $93(12.2)$ & $153(20.0)$ & $23(3.0)$ & $80(10.5)$ & $6(0.8)$ & $765(100)$ \\
\hline
\end{tabular}

Table 5. Initial result vs. worst colonoscopic result after initial colonoscopy amongst late onset FCC families

\begin{tabular}{llllllll} 
& \multicolumn{7}{c}{ Worst follow-up result } \\
\cline { 2 - 7 } Worst baseline result & Normal & $\begin{array}{l}\text { Hyperplastic } \\
\text { Polyp }\end{array}$ & Adenoma & $\begin{array}{l}\text { Multiple } \\
\text { adenomas }\end{array}$ & $\begin{array}{l}\text { High Risk } \\
\text { adenoma }\end{array}$ & Cancer & Total \\
\hline Normal & $100(64.9)$ & $17(11.0)$ & $26(16.9)$ & $4(2.6)$ & $7(4.5)$ & - & $154(100)$ \\
Hyperplastic Polyp & $17(40.5)$ & $11(26.2)$ & $8(19.0)$ & - & $5(11.9)$ & $1(2.4)$ & $42(100)$ \\
Adenoma & $22(40.0)$ & $9(16.4)$ & $17(30.9)$ & $1(1.8)$ & $6(10.9)$ & - & $55(100)$ \\
Multiple adenomas & $1(25.0)$ & - & $1(25.0)$ & - & $1(25.0)$ & $1(25.0)$ & $4(100)$ \\
High risk adenoma & $11(33.3)$ & $3(9.1)$ & $5(15.2)$ & $2(6.1)$ & $11(33.3)$ & $1(3.0)$ & $33(100)$ \\
Total & $151(52.4)$ & $40(13.9)$ & $57(19.8)$ & $7(2.4)$ & $30(10.4)$ & $3(1.0)$ & $288(100)$ \\
\hline
\end{tabular}

Numbers shown are $n$ (percentage)

individuals from type $\mathrm{X}$ families was slightly younger than those from LOFCC families (51 years vs. 54 years). Adjusting for age, the results were similar for those families classified by tumor and by germline genetic testing. There was a slightly higher proportion of normal colonoscopy in individuals from FCC type X than LOFCC families (66.8\% vs. $57.6 \%$ respectively, $p<0.0001$ ) (Table 3 ).

\section{Findings at follow-up surveillance colonoscopy}

Of the 1,541 individuals who were not diagnosed with CRC at baseline colonoscopy, 1,088 (70.6\%) had at least one fur- ther colonoscopy of whom 1,053 (96.8\%) had at least one result recorded. The worst (most advanced pathology) result on follow-up relative to the baseline colonoscopy result is shown in Tables (4-6). It is seen that the results for FCC type $\mathrm{X}$ and LOFCC are similar (Tables 4 and 5). In the cohort as a whole (Table 6) over a third of individuals with either a high-risk adenoma or multiple adenomas at baseline had a high-risk adenoma or cancer on follow-up. If we considered a more established definition of multiple adenomas (i.e., three or more adenomas on any one colonoscopy or five or more adenomas throughout the colonoscopic surveillance) 
Table 6. Initial result vs. worst colonoscopic result after initial colonoscopy amongst all families

\begin{tabular}{llllllll}
\hline & \multicolumn{7}{c}{ Worst follow-up result } \\
\cline { 2 - 7 } Worst baseline result & Normal & $\begin{array}{l}\text { Hyperplastic } \\
\text { Polyp }\end{array}$ & Adenoma & $\begin{array}{l}\text { Multiple } \\
\text { adenomas }\end{array}$ & $\begin{array}{l}\text { High Risk } \\
\text { adenoma }\end{array}$ & Cancer & Total \\
\hline Normal & $426(64.1)$ & $71(10.7)$ & $117(17.6)$ & $10(1.5)$ & $39(5.9)$ & $2(0.3)$ & $665(100)$ \\
Hyperplastic Polyp & $36(30.8)$ & $36(30.8)$ & $22(18.8)$ & $7(6.0)$ & $14(12.0)$ & $2(1.7)$ & $117(100)$ \\
Adenoma & $60(37.7)$ & $19(12.0)$ & $53(33.3)$ & $8(5.0)$ & $18(11.3)$ & $1(0.6)$ & $159(100)$ \\
Multiple adenomas & $4(20.0)$ & $2(10.0)$ & $2(10.0)$ & $2(10.0)$ & $7(35.0)$ & $3(15.0)$ & $20(100)$ \\
\hline High risk adenoma & $35(38.0)$ & $5(5.4)$ & $16(17.4)$ & $3(3.3)$ & $32(34.8)$ & $1(1.1)$ & $92(100)$ \\
Total & $561(53.3)$ & $133(12.6)$ & $210(19.9)$ & $30(2.9)$ & $110(10.5)$ & $9(0.9)$ & $1.053(100)$ \\
\hline
\end{tabular}

Numbers shown are $n$ (percentage).

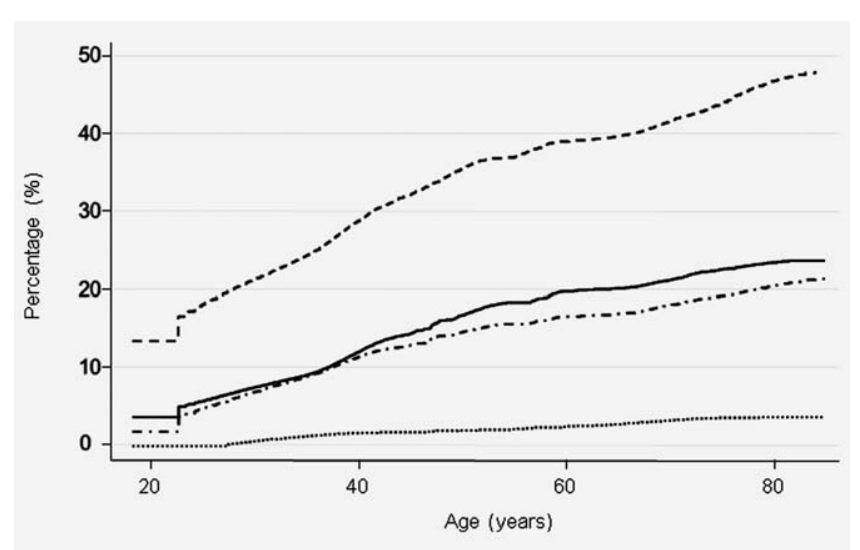

Figure 1. Cumulative proportion of colonoscopic surveillance outcomes according to age. Individuals are censored at the first event. The proportion of colonoscopies with an event is estimated by smoothing and is forced to be non-decreasing in age by applying the pool adjacent violators algorithm. - - - - Adenoma or worse, — Multiple Adenoma or worse, - . - .- . high-rish Adenoma or worse, ............ Colorectal cancer.

then there were 14 more individuals defined as having multiple adenomas. None of these individuals went on to subsequently develop a high-risk adenoma or cancer. The lowest risk of advanced neoplasia on surveillance was in those with a normal baseline examination (6\% had a high-risk adenoma or worse). The risk of a subsequent high-risk adenoma or worse was similar in those with a hyperplastic polyp (13.7\%) or an adenoma (11.9\%) on baseline.

The cumulative proportions of individuals with increasing age having a pathological finding on colonoscopy are shown in Figure 1. Some adenomas are detected on initial colonoscopy (from approximately age 25 years) with a steady increase to just over $30 \%$ of individuals affected at 40 years of age. The incidence of multiple adenomas and high-risk adenomas increases after 40 years of age to 80 years of age from approximately $10 \%$ to just over $20 \%$.

A total of 1,513 adenomas were identified from all individuals included in the study. Of these, 390 (26\%) were classified as high-risk adenomas. The risk of having a high-risk adenoma was higher in individuals with a history of multiple adenomas compared to those who had adenomas that were not classified as multiple (53\% and 33\% respectively); however the proportion of adenomas which were classified as high-risk was slightly lower in the multiple adenoma group ( $21 \%$ vs. $29 \%)$. Data were collected on the distribution of adenomas from Denmark, The Netherlands, Manchester, Stockholm and London. From these five centers, a total of 465 adenomas were identified at the baseline colonoscopy. Eleven adenomas (2.4\%) were from an unknown location. Of the remaining 454 adenomas, 248 (55\%) were from the left side, $183(40 \%)$ from the right side and $23(5.1 \%)$ were identified along with other adenomas on the same colonoscopy and records showed adenomas from left and right side of the colon. Throughout the total follow-up, 811 adenomas were identified (31 unknown location); 352 (45\%) from the left side, 366 (47\%) from the right side and 62 (7.9\%) from both the left and right side.

To determine whether the multiple adenoma phenotype clustered within FCC families, the presence or absence of multiple adenomas was assessed within families with at least two members undergoing surveillance. In 55/999 (5.5\%) of individuals, only one family member had multiple adenomas. In 23/347 (6.6\%) of cases, multiple adenomas were also seen in another family member giving a relative risk of $1.2(95 \%$ CI 0.8-1.6).

The proportion of individuals under surveillance developing CRC remains low across all ages. In total, nine CRCs were identified during surveillance. Details of these cancers are shown in Table 7 . The time interval since the previous colonoscopy ranged from 2 to 6 years, (median 2.7 years). The ages of individuals at the time of their cancer diagnosis ranged from 42 to 77 years.

\section{Discussion}

The results of a pooled multicenter comparative study of the outcome of prospective colonoscopic surveillance in FCC have been presented. Data have been collected for up to more than 30 years and demonstrate that there is a high prevalence of adenomas and CRC in the population studied, the incidence of adenomas and advanced neoplasia is increased compared to the general population, and that 


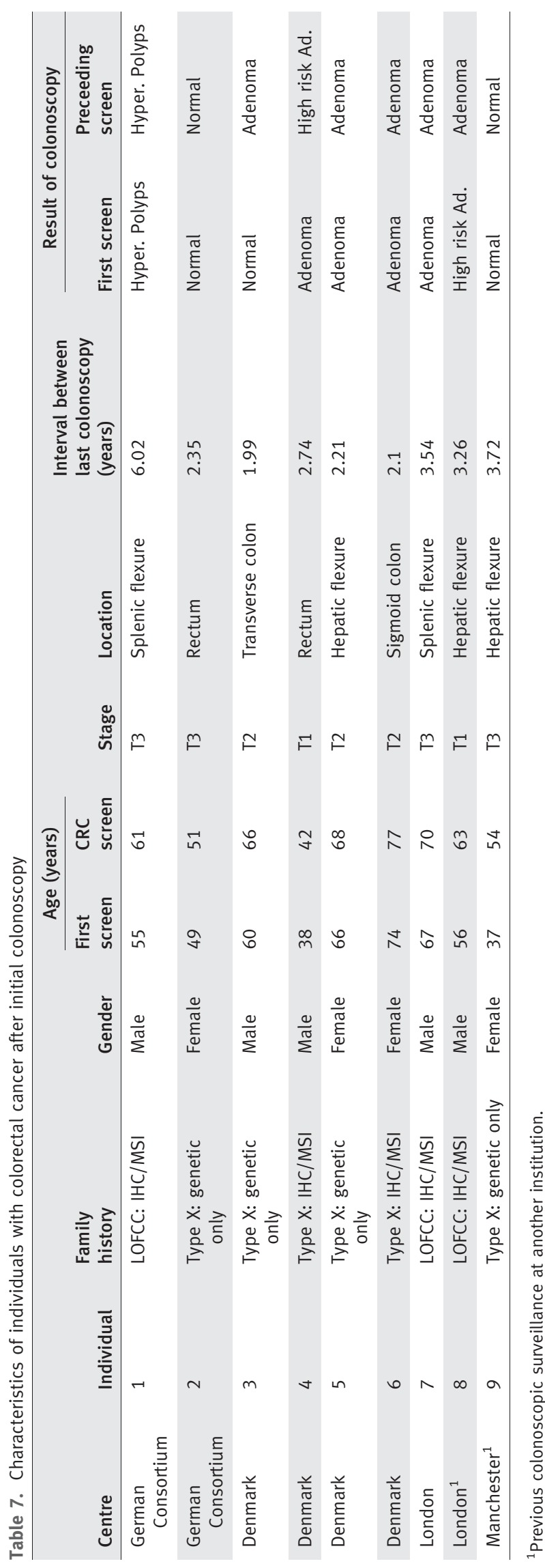

interval cancers do occur during surveillance although they may be detected at an early stage. There was no obvious difference in phenotype during colonoscopic surveillance between FCC type X and LOFCC and these should be managed as a single group.

Baseline colonoscopy results confirmed that FCC individuals have an increased prevalence of colonic neoplasia. ${ }^{11}$ The median age at baseline colonoscopy was 47.5 years. At baseline, $21.8 \%$ of individuals had one or more adenomas, including 7.6\% with high-risk adenomas, and $1.4 \% \quad(n=22)$ asymptomatic cancers with a median age of 52 (range 3478).The (standardized prevalence) ratio for CRC was significantly raised compared to the population of England.

We have analyzed the incidence of adenomas, high-risk adenomas and multiple adenomas by age in Figure 1 (censoring when an adenoma has been removed at colonoscopy). As expected, there is an increasing incidence with age, and we have shown that approximately $40 \%$ of individuals have at least one adenoma, $20 \%$ multiple adenomas and $18 \%$ a high risk adenoma by age 70 years. A similar trend is seen in average risk individuals but with a much lower frequency. ${ }^{11,12}$ Cancers occurred more frequently in individuals with multiple adenomas. Our definition of individuals with multiple adenomas was introduced to avoid bias with varying duration and frequency of colonoscopic surveillance. In individuals classified as having multiple adenomas, the risk of an individual adenoma being high-risk was not increased although there was an increased risk of an individual having a highrisk adenoma. We were not able to demonstrate significant clustering of multiple adenomas within families. Overall in baseline and follow-up colonoscopies, adenomas were fairly evenly distributed between the left and right colon $(52 \% v s$. $48 \%$ respectively).

During follow-up surveillance, the youngest age at which a cancer was diagnosed was 42 and the eldest 77 with a median age of 63 years. It is notable that five cancers occurred within three years of a previous colonoscopy although four of these cancers were detected at an early stage (T1 or T2). In four of these cases, an adenoma was present at the previous colonoscopy. There is a well-described miss rate of significant pathology even with close colonoscopic surveillance. ${ }^{12}$ In our analysis, we have assumed that pathology detected on follow-up colonoscopy has developed since the previous examination although, in fact, some of these may represent neoplasia that was previously missed. Six of the cancers were in the right side of the colon and three were in the left colon. Lindor et al. report that in FCC type X pedigrees the cancers were predominantly left-sided. ${ }^{3}$ A higher proportion of the interval cancers are right-sided than the cancers diagnosed at baseline colonoscopy (67\% vs. $26 \%$ respectively, $p=0.017)$. There are reports that colonoscopic surveillance in the general population is much less effective at preventing right-sided than left-sided sided cancers and this may also apply to FCC individuals. ${ }^{13}$ This result emphasizes the importance of particularly careful inspection of the 
right colon at colonoscopy, and that a repeat colonoscopy/ alternative procedure should be carried out if the examination is not complete with good views to the cecum.

Despite these nine cases, the incidence of CRC in this study was very low at 1.14 per 1,000 person years. This compares very favorably to 15.8 cancers per 1,000 person years in individuals with LS undergoing surveillance previously reported in the control arm of the CAPP2 study. ${ }^{14}$ There may also be a role for aspirin chemoprevention in FCC which has been demonstrated to have a protective effect in randomized studies of individuals at population risk and also in individuals with LS. ${ }^{14,15}$

It is possible that our cohort of patients included families with LS, especially since several cancers were diagnosed within 3 years of colonoscopy raising the possibility of an accelerated adenoma-carcinoma pathway. LS was excluded either by testing for loss of expression of MLH1 and MSH2 proteins using immunohistochemistry, the presence of DNA MSI in one or more CRCs from an affected family member and/or undertaking germline sequencing of two ( $M L H 1$ and MSH2) or more DNA mismatch repair genes for alterations (see Table 2). Mutations in $M L H 1$ and $M S H 2$ are responsible for the majority of cases of LS but mutation detection techniques are imperfect. Approximately, 6\% of cases of LS are due to MSH6 mutations and 2\% to PMS2 mutations. Immunohistochemistry and/or germline sequencing for MSH6 and PMS2 was not undertaken in all families. We have reviewed in detail the genetic testing that had been undertaken in the nine cancers that were diagnosed in the cohort during surveillance (Table 7). In five cases, tumors from several family members had been tested for DNA MSI and germline sequencing also undertaken. We have also looked at the incidence of extra-colonic cancers associated with LS in our families and found no increase. This suggests that our findings of advanced neoplasia on colonoscopic surveillance are not due to undetected LS families being included in the cohort.

This pooled analysis confirms that individuals from families in which there is a dominant pattern of CRC inheritance, but no evidence of DNA mismatch repair deficiency, are at high risk of subsequently developing adenomas and CRC. This is likely to be a genetically heterogeneous group. One interesting finding from this study is that there was no obvious difference in the colonoscopic findings of individuals from FCC type X or LOFCC families and for this reason, we have grouped them together. It is likely that they both represent a similar spectrum of genetic predispositions. Genomewide linkage studies have been undertaken in families with a multiple adenoma phenotype and have demonstrated linkage to various loci including 3q 21-24 and q21.1-26.2 and 9q22$33,{ }^{16-18}$ and a small proportion of cases may be due to attenuated alterations of the APC gene. However, germline mutations responsible for FCC have, by and large, not been identified. Whole genome sequencing is now being used to investigate individuals with multiple adenomas and may well reveal novel genetic alterations predisposing to this phenotype. ${ }^{19}$ FCC may be due to a complex genetic model of dominant genes, rare variants and low penetrance variants.

One of the strengths of this study is that each center has a slightly different surveillance protocol (see Table 1). There is variation in the age at which colonoscopic surveillance is started, the intervals between examinations and whether examinations are carried out in local hospitals or tertiary referral centers. This reflects the breadth of clinical practice in Europe and therefore provides a robust picture of the effectiveness of colonoscopic surveillance in this high-risk group. The number of prevalent cancer cases in this study is compared to CRC cases in the general population in England ${ }^{9}$ (rather than each country's data) which is a weakness of the study. The incidence in England is very similar to other European countries included in this analysis although slightly lower than Sweden and The Netherlands. ${ }^{20}$ However, the higher incidence in these countries is not sufficient to explain the high prevalence of CRC cases compared to the general population of England.

Although multiple adenomas did subsequently develop in individuals with a normal baseline colonoscopy, interval cancers predominantly occurred in individuals who had already developed multiple adenomas. The incidence of adenomas and cancers increases with age. As might be anticipated if there is an autosomal dominant predisposition, $40 \%$ of atrisk individuals never developed any type of polyp. These results support the idea that colonoscopic surveillance might be undertaken less frequently at a younger age and in those that have not developed adenomas, whereas those who have developed high-risk or multiple adenomas should undergo more frequent colonoscopic surveillance (as is the current practice).

In conclusion, there is no obvious difference in the outcome of colonoscopic surveillance between FCC type X, in which a family member had developed CRC before the age of 50 years, and LOFCC, in which there were no cases of CRC under the age of 50 years. In FCC there is a significantly increased prevalence of CRC and a significantly increased incidence of adenomas and advanced adenomas on prospective colonoscopic surveillance compared to published studies of average risk individuals of similar age. However, there is a much lower incidence of interval cancers than seen on colonoscopic surveillance in the CAPP2 study of LS. ${ }^{14}$ The frequency of adenomas increases with age and uncommon interval cancers occur predominantly at a later age and in individuals who have developed multiple adenomas. However, we estimate a high cumulative proportion of individuals with a high-risk adenoma at age 40 of approximately $10 \%$. We suggest that colonoscopic surveillance with five yearly intervals should commence between 30 and 40 years of age although there is little evidence to suggest screening before this age. Colonoscopic frequency should be increased if adenomas are found according to current adenoma follow-up guidelines. $^{1}$ 


\section{Acknowledgements}

The authors would like to thank the Mallorca Group for the forum to initiate this European collaboration and their thoughts and suggestions during the development. They would like to thank Maggie Stevens, Carole Cummings, Julie Jefferies and Elizabeth Goodband for their administrative support at St Mark's and Kate Green for data management in Manchester. Authors thank Polly Rawlinson for administrative input with the manuscript. DGE and FL are supported by the Manchester BRC.

\section{Collaborators "German HNPCC Consortium"}

\begin{tabular}{llll}
\hline First name & Middle initial & Last name & Center \\
\hline Christiane & & Bernhardt & Bochum \\
Joerg & T. & Epplen & Bochum \\
Johanna & & Munding & Bochum \\
Christian & Pox & Bochum \\
Wolff & Schmiegel & Bochum \\
Karsten & Schulmann & Bochum \\
Susanne & Stemmler & Bochum \\
Andrea & & Tannapfel & Bochum \\
Markus & Noethen & Bonn \\
Peter & Propping & Bonn \\
Verena & & Steinke & Bonn \\
Reinhard & & Buettner & Cologne \\
Philip & & Kahl & Cologne \\
Daniela & E. & Aust & Dresden \\
Heike & & Goergens & Dresden \\
\hline
\end{tabular}

(Continued)
(Continued)

\begin{tabular}{|c|c|c|c|}
\hline Barbara & & Klink & Dresden \\
\hline Steffen & & Pistorius & Dresden \\
\hline Hans & $\mathrm{K}$. & Schackert & Dresden \\
\hline Timm & & Goecke & Duesseldor \\
\hline Olivia & & Kiehl & Duesseldor \\
\hline Gabriela & & Moeslein & Duesseldor \\
\hline Nils & & Rahner & Duesseldor \\
\hline Christina & & Evers & Heidelberg \\
\hline Monika & & Keller & Heidelberg \\
\hline Matthias & & Kloor & Heidelberg \\
\hline Mirjam & & Tariverdian & Heidelberg \\
\hline Magnus & & vonKnebelDoeberitz & Heidelberg \\
\hline Ute & & Enders & Leipzig \\
\hline Christoph & & Engel & Leipzig \\
\hline Marlies & & Herold & Leipzig \\
\hline Markus & & Loeffler & Leipzig \\
\hline Kerstin & & Wieland & Leipzig \\
\hline Silke & & Zachariae & Leipzig \\
\hline Monika & & Morak & Munich \\
\hline Gisela & & Keller & Munich \\
\hline Stefan & & Dechant & Regensburg \\
\hline Wolfgang & & Dietmaier & Regensburg \\
\hline Petra & & Ruemmele & Regensburg \\
\hline
\end{tabular}

\section{References}

1. Cairns SR, Scholefield JH, Steele RJ, et al. Guidelines for colorectal cancer screening and surveillance in moderate and high risk groups (update from 2002). Gut 2010;59:666-89.

2. Vasen HF, Mecklin JP, Khan PM, et al. The International Collaborative Group on Hereditary Non-Polyposis Colorectal Cancer (ICG-HNPCC) Dis Colon Rectum 1991;34:424-5.

3. Lindor NM, Rabe K, Petersen GM, et al. Lower cancer incidence in Amsterdam-I criteria families without mismatch repair deficiency: familial colorectal cancer type X. JAMA 2005;293:1979-85.

4. Lindgren G, Liljegren A, Jaramillo E, et al. Adenoma prevalence and cancer risk in familial nonpolyposis colorectal cancer. Gut 2002;50:228-34

5. Dove-Edwin I, de Jong AE, Adams J, et al. Prospective results of surveillance colonoscopy in dominant familial colorectal cancer with and without Lynch syndrome. Gastroenterology 2006; 130:1995-2000.

6. Engel C, Rahner N, Schulmann K, et al. Efficacy of annual colonoscopic surveillance in individuals with hereditary nonpolyposis colorectal cancer. Clin Gastroenterol Hepatol 2010;8:174-82.

7. Vasen HF, Abdirahman M, Brohet R, et al. One to 2-year surveillance intervals reduce risk of colorectal cancer in families with Lynch syndrome. Gastroenterology 2010;138:2300-6.
8. Atkin WS, Edwards R, Kralj-Hans I, et al. Onceonly flexible sigmoidoscopy screening in prevention of colorectal cancer: a multicentre randomised controlled trial. Lancet 2010;375:1624-33.

9. Cancer Statistics Registrations, England (Series MB1), No. 40, 2009. Available at: http:// www.ons.gov.uk/ons/rel/vsob1/cancer-statisticsregistrations-england-series-mb1-/no-40-2009/ index.html]. Last accessed [September 2012].

10. Robertson T, Wright FT, Dykstra RL. Order restricted statistical inferenceed. Chichester: Wiley, 1988.

11. Imperiale TF, Wagner DR, Lin CY, et al. Results of screening colonoscopy among persons 40 to 49 years of age. N Engl J Med 2002;346:1781-5.

12. Robertson DJ, Greenberg ER, Beach M, et al. Colorectal cancer in patients under close colonoscopic surveillance. Gastroenterology 2005;129:3441.

13. Singh $H$, Nugent $Z$, Demers AA, et al. The reduction in colorectal cancer mortality after colonoscopy varies by site of the cancer. Gastroenterology 2010;139:1128-37.

14. Burn J, Gerdes AM, Macrae F, et al. Long-term effect of aspirin on cancer risk in carriers of hereditary colorectal cancer: an analysis from the CAPP2 randomised controlled trial. Lancet 2011; 378:2081-7.
15. Rothwell PM, Fowkes FG, Belch JF, et al. Effect of daily aspirin on long-term risk of death due to cancer: analysis of individual patient data from randomised trials. Lancet 2011; 377:31-41.

16. Kemp Z, Carvajal-Carmona L, Spain S, et al. Evidence for a colorectal cancer susceptibility locus on chromosome 3q21-q24 from a highdensity SNP genome-wide linkage scan. Hum Mol Genet 2006;15:2903-10.

17. Picelli S, Vandrovcova J, Jones S, et al. Genomewide linkage scan for colorectal cancer susceptibility genes supports linkage to chromosome $3 q$. BMC Cancer 2008;8:87.

18. Wiesner GL, Daley D, Lewis S, et al. A subset of familial colorectal neoplasia kindreds linked to chromosome 9q22.2-31.2. Proc Natl Acad Sci USA 2003;100:12961-5.

19. Palles C, Cazier JB, Howarth KM, et al. Germline mutations affecting the proofreading domains of POLE and POLD1 predispose to colorectal adenomas and carcinomas. Nat Genet 2012;45: 136-44.

20. Estimated incidence, mortality \& prevalence in men Cancer of the large bowel Lyon: IARC/ EUCAN, 2012. Avaialble at: http://eco.iarc.fr/ eucan/cancer.aspx? cancer $=10]$. Last accessed [June 2013]. 